\title{
ERRATUM
}

\section{Erratum to: Effect of biaxial strain and hydrostatic pressure on the magnetic properties of bilayer $\mathrm{CrI}_{3}$}

\author{
Chong $\mathrm{Xu}^{1}$, Qian-Jun Wang ${ }^{1}$, Bin $\mathrm{Xu}^{1}$, Jun $\mathbf{H u}^{1,2, \dagger}$ \\ ${ }^{1}$ School of Physical Science and Technology \& Jiangsu Key Laboratory of Thin Films, Soochow University, \\ Suzhou 215006, China \\ ${ }^{2}$ School of Physical Science and Technology, Ningbo University, Ningbo 315211, China \\ Corresponding author. E-mail: ${ }^{\dagger}$ hujun2@nbu.edu.cn \\ Received May 14, 2021; accepted May 18, 2021
}

ERRATUM TO: Front. Phys. 53502 (2021), https://doi. org/10.1007/s11467-021-1073-x

The latest version of the original publication of this article is attached. The online version of the original article can be found at https://doi.org/10.1007/s11467021-1073-x and http://journal.hep.com.cn/fop/EN/10. 1007/s11467-021-1073-x.

\footnotetext{
${ }^{*}$ This article can also be found at http://journal.hep.com. cn/fop/EN/10.1007/s11467-021-1083-8.
} 


\title{
Effect of biaxial strain and hydrostatic pressure on the magnetic properties of bilayer $\mathrm{CrI}_{3}$
}

\author{
Chong $\mathrm{Xu}^{1}$, Qian-Jun Wang ${ }^{1}$, Bin $\mathrm{Xu}^{1}$, Jun $\mathbf{H u}^{1,2, \dagger}$ \\ ${ }^{1}$ School of Physical Science and Technology $\&$ Jiangsu Key Laboratory of Thin Films, \\ Soochow University, Suzhou 215006, China \\ ${ }^{2}$ School of Physical Science and Technology, Ningbo University, Ningbo 315211, China \\ Corresponding author. E-mail: ${ }^{\dagger}$ hujun2@nbu.edu.cn \\ Received January 19, 2021; accepted April 8, 2021
}

\begin{abstract}
Two-dimensional van der Waals magnetic materials are intriguing for applications in the future spintronics devices, so it is crucial to explore strategy to control the magnetic properties. Here, we carried out first-principles calculations and Monte Carlo simulations to investigate the effect of biaxial strain and hydrostatic pressure on the magnetic properties of the bilayer $\mathrm{CrI}_{3}$. We found that the magnetic anisotropy, intralayer and interlayer exchange interactions, and Curie temperature can be tuned by biaxial strain and hydrostatic pressure. Large compressive biaxial strain may induce a ferromagneticto-antiferromagnetic transition in both $\mathrm{CrI}_{3}$ layers. The hydrostatic pressure could enhance the intralayer exchange interaction significantly and hence largely boost the Curie temperature. The effect of the biaxial strain and hydrostatic pressure revealed in the bilayer $\mathrm{CrI}_{3}$ may be generalized to other two-dimensional magnetic materials.
\end{abstract}

Keywords bilayer $\mathrm{CrI}_{3}$, biaxial strain, hydrostatic pressure, magnetic properties

\section{Introduction}

Van der Waals two-dimensional (2D) materials have evoked great interest of researchers in the community of condensed matter physics and materials science, since the discovery of graphene [1], because they exhibit abundant new physical properties, and are promising in various applications in electronic and optoelectronic devices [2]. Recently, a new class of $2 \mathrm{D}$ materials $-2 \mathrm{D}$ ferromagnetic (FM) materials - have been discovered [3, 4]. The 2D FM materials are particularly interesting not only in the aspect of fundamental science, because it was unexpected according to the Mermin-Wagner theorem [5], but also in the development of new generation of spintronics devices, because the highest Curie temperature $\left(T_{\mathrm{C}}\right)$ may even achieve to room temperature [6-9].

The bulk $\mathrm{CrI}_{3}$ is FM with the $T_{\mathrm{C}}$ of $61 \mathrm{~K}$ and a rhombohedral layer stacking $[10,11]$. The FM ordering persists in few-layer $\mathrm{CrI}_{3}$ except bilayer, with the $T_{\mathrm{C}}$ down to $45 \mathrm{~K}$ for the monolayer $\mathrm{CrI}_{3}$, whereas the bilayer $\mathrm{CrI}_{3}$ displays interlayer antiferromagnetic (AFM) coupling [4]. It is known that the physical properties may be closely associated with the stacking order in 2D matertials [12].

\footnotetext{
*arXiv: 2104.13525. This article can also be found at http://journal.hep.com.cn/fop/EN/10.1007/s11467-021$1073-\mathrm{x}$.
}

Indeed, a series of literatures have reported that the interlayer magnetic coupling in the bilayer $\mathrm{CrI}_{3}$ depends on the stacking pattern $[13-16]$. With the AB-stacking order [Fig. 1(a)] where the top layer laterally shifts by $\left[\frac{2}{3}, \frac{1}{3}\right]$ in fractional coordinates with respect to the bottom layer, the two $\mathrm{CrI}_{3}$ layers couple ferromagnetically. With the $\mathrm{AB}^{\prime}$-stacking order [Fig. 1(b)] where the lateral shift is $\left[\frac{1}{3}, \frac{1}{3}\right]$, the two $\mathrm{CrI}_{3}$ layers couple antiferromagnetically. In fact, these two types of stacking orders correspond to the bulk $\mathrm{CrI}_{3}$ at low temperature (below $210 \mathrm{~K}$ ) with the space group $R \overline{3}$ and room temperature with the space group $C 2 / m$, respectively [11, 17]. Nevertheless, the $\mathrm{AB}^{\prime}$ stacking bilayer $\mathrm{CrI}_{3}$ was usually observed in experiment, probably because the bilayer exfoliated at room temperature is kinetically trapped in the $\mathrm{AB}^{\prime}$-stacking order during the cooling process [13]. Interestingly, the interlayer magnetism of the bilayer $\mathrm{CrI}_{3}$ can be tuned by electric field, gate voltage and external pressure [17-21].

Magnetic anisotropy (MA) is an important requirement for realizing long-range magnetic ordering in $2 \mathrm{D}$ magnetic materials $[4,22]$, because the long-range $2 \mathrm{D}$ magnetic ordering cannot establish in the spin-rotational invariant systems as demonstrated in the Mermin-Wagner theorem [5]. Magnetic anisotropy plays the role of breaking the spin rotational invariance, and thus allows the long-range 2D magnetic ordering to exist. For the sake of practical applications in spintronics devices, perpendicular magnetic anisotropy (PMA) and FM ordering at 
(a)
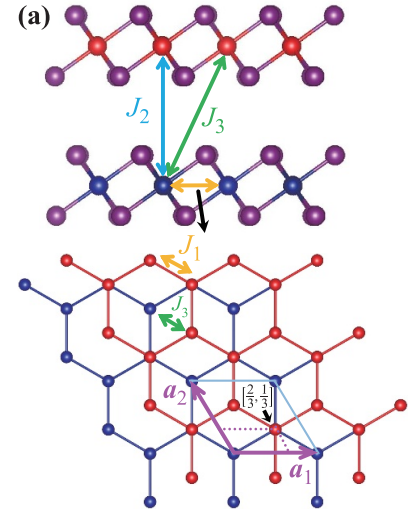

(b)
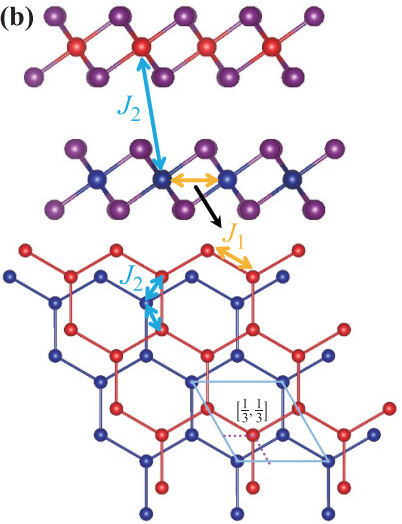

Fig. 1 Side and top views of the atomic structure of bilayer $\mathrm{CrI}_{3}$ with (a) AB-stacking order and (b) $\mathrm{AB}^{\prime}$-stacking order. The red and blue spheres stand for the Cr atoms in the top and bottom layers, respectively. The purple spheres stand for the I atoms. The intralayer $\left(J_{1}\right)$ and interlayer $\left(J_{2}\right.$ and $\left.J_{3}\right)$ exchange parameters are indicated. In the bottom layers, only the $\mathrm{Cr}$ atoms are shown, and the light blue parallelograms denote the unit cells of the AB- and $\mathrm{AB}^{\prime}$-stacking bilayers. The purple arrows mark the lattice vectors, and the purple dotted lines indicate the lateral shift (in fractional coordinates) of the top layer (red) with respect to the bottom layer.

room temperature are required simultaneously. Therefore, strong PMA and FM ordering are desired and it is important to explore strategies to control the MA and to enhance $T_{\mathrm{C}}[23]$. Although theoretical study of the effect of strain on the MA of the monolayer $\mathrm{CrI}_{3}$ has been done recently [24-27], systematic theoretical investigation of the electronic and magnetic properties including the $\mathrm{MA}$ and magnetic ordering of the bilayer $\mathrm{CrI}_{3}$ under strain is still lacking [28].

In this paper, we explored the effect of biaxial strain and hydrostatic pressure on the electronic and magnetic properties of the bilayer $\mathrm{CrI}_{3}$, based on first-principles calculations and Monto Carlo simulation. We found that the magnetic properties of the bilayer $\mathrm{CrI}_{3}$ are sensitive to biaxial strain and hydrostatic pressure. In particular, the hydrostatic pressure may turn the easy axis from the outof-plane direction (i.e., PMA) to in-plane direction. The intralayer exchange interaction may be significantly enhanced by the hydrostatic pressure, which may lead to $T_{\mathrm{C}}$ over $100 \mathrm{~K}$, much higher than the original $T_{\mathrm{C}}$ without the hydrostatic pressure.

\section{Computational details}

The atomic structures, electronic and magnetic properties were calculated by using the Vienna ab-initio simulation package in the framework of the density functional theory (DFT) $[29,30]$. The interaction between valence electrons and ionic cores was described within the framework of the projector augmented wave (PAW) method [31, 32]. Van der Waals interaction was included with the optB86b exchange functional $[33,34]$. Hubbard $U$ of $3 \mathrm{eV}$ for the $\mathrm{Cr}$ atom was adopted to account for the strong electronic correlations [35]. The energy cutoff for the plane wave basis expansion was set to $650 \mathrm{eV}$. The two-dimensional Brillouin zone was sampled by a $24 \times 24 \mathrm{k}$-grid mesh. The atomic positions were fully relaxed using the conjugated gradient method until the force on each atom is smaller than $0.01 \mathrm{eV} / \AA$.

The exchange coupling parameters of the bilayer $\mathrm{CrI}_{3}$ can be extracted by comparing the total energies with different spin configurations (see Fig. 2), based on the Heisenberg spin Hamiltonian

$$
H=-\sum_{i, j} J_{i j} \boldsymbol{S}_{i} \cdot \boldsymbol{S}_{j} .
$$

As illustrated in Fig. 1, we considered the first nearest neighboring intralayer exchange interaction (denoted by $J_{1}$ ) for both $\mathrm{AB}$ - and $\mathrm{AB}^{\prime}$-stacking bilayers. For the interlayer couplings, the dominant exchange interactions can be represented by $J_{2}$ and $J_{3}$ for the AB-stacking bilayer $\mathrm{CrI}_{3}$ [Fig. 1(a)] and $J_{2}$ for the $\mathrm{AB}^{\prime}$-stacking bilayer $\mathrm{CrI}_{3}$ [Fig. 1(b)]. With these exchange interaction parameters, we performed Monte Carlo simulations with a $64 \times 64$ supercell to estimate the $T_{\mathrm{C}}$.

\section{Results and discussion}

We started our calculations from the experimental lattice constant of the bulk $\mathrm{CrI}_{3}\left(a_{0}=6.867 \AA\right)$, and then applied in-plane biaxial strain $(\varepsilon)$ from $-6 \%$ to $6 \%$. The negative and positive signs indicate compressive and extensile strains, respectively. The corresponding lattice constant varies from $6.45 \AA$ to $7.28 \AA$. Accordingly, the distance between the nearest $\mathrm{Cr}$ atoms increases from $3.73 \AA$ to $4.20 \AA$, and the $\mathrm{Cr}-\mathrm{I}$ bond lengths increase from $2.71 \AA$ to $2.79 \AA$, as plotted in Fig. 3(a). Clearly, the variation of the $\mathrm{Cr}-\mathrm{I}$ bond lengths (from $-1.45 \%$ and $1.45 \%$ ) is much smaller than the in-plane biaxial strain, because the outof-plane positions of the $\mathrm{Cr}$ and I atoms are free to relax. Consequently, the $\mathrm{Cr}-\mathrm{I}-\mathrm{Cr}$ angle $(\alpha)$ increases significantly when the strain varies from $-6 \%$ to $6 \%$, as shown in Fig. 3(b). There are two types of $\mathrm{I}-\mathrm{Cr}-\mathrm{I}$ angles, the angle formed by one $\mathrm{Cr}$ atom and two I atoms lying in the same atomic plane (two atomic planes) is denoted as $\theta_{1}\left(\theta_{2}\right)$. $\theta_{1}$ increases but slower than $\alpha$, while $\theta_{2}$ decreases significantly, as seen in Fig. 3(b). Note that these structural parameters do not depend on the stacking patterns.

The bilayer $\mathrm{CrI}_{3}$ is semiconducting with the band gap of 1.01 and $1.07 \mathrm{eV}$, respectively for the $\mathrm{AB}$ - and $\mathrm{AB}^{\prime}$ stacking bilayers, slightly smaller than that of the monolayer $\mathrm{CrI}_{3}(1.09 \mathrm{eV})$. Generally speaking, the band gap of a semiconductor will increase (decrease) under compressive (extensile) strain, as sketched in Fig. 4(a). However, the band gap of the bilayer $\mathrm{CrI}_{3}$ exhibits opposite trend. As shown in Figs. 4(c) and (d), the band gaps of 


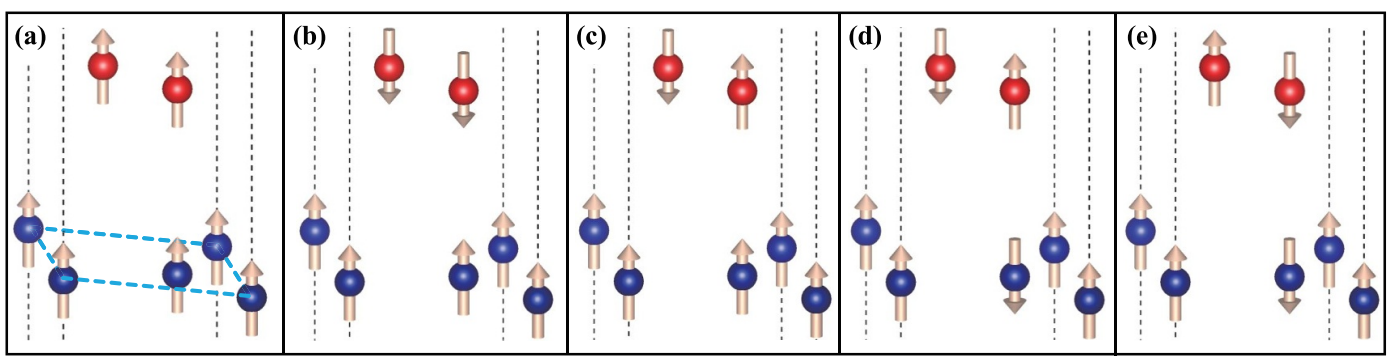

Fig. 2 Magnetic configurations of AB-stacking bilayer $\mathrm{CrI}_{3}$. The dashed parallelogram denotes the in-plane unit cell. (a) Intralayer and interlayer ferromagnetism. (b) Intralayer ferromagnetism and interlayer antiferromagnetism. (c) Interlayer ferrimagnetism. The bottom $\mathrm{CrI}_{3}$ layer is ferromagnetic, while the top $\mathrm{CrI}_{3}$ layer is antiferromagnetic. (d) Intralayer and interlayer antiferromagnetism. (e) Intralayer antiferromagnetism and interlayer ferromagnetism. The magnetic configurations of $\mathrm{AB}^{\prime}$-stacking bilayer $\mathrm{CrI}_{3}$ are similar.

the $\mathrm{AB}$ - and $\mathrm{AB}^{\prime}$-stacking bilayers respectively decrease to 0.74 and $0.69 \mathrm{eV}$ under compressive strain of $-6 \%$, while they increase to 1.13 and $1.22 \mathrm{eV}$ under extensile strain of $6 \%$. This abnormal phenomenon can be explained by the feature of the local structure which is represented by the distorted octahedron formed by one $\mathrm{Cr}$ atom and six I atoms as displayed in Fig. 4(b). In the local coordinate frame, the $3 \mathrm{~d}$ orbitals of the $\mathrm{Cr}$ atom split into the triplet $t_{2 g}$ orbital and the doublet $e_{g}$ orbital due to the crystal field, as seen from the inset in Fig. 4(c). The band gap lies between the occupied $t_{2 g}$ states and the empty $e_{g}$ states, and thus is primarily determined by the crystal-field splitting. When a compressive (extensile) biaxial strain is applied onto the unit cell, the equivalent strain onto the octahedron is actually extensile (compressive) along the $\mathrm{C}_{3}$ axis as indicated in Fig. 4(b). Therefore, the compressive (extensile) biaxial strain leads to the octahedron to be stretched (compressed), and thus weakens (strengthens) the crystal-field splitting. Consequently, the energy levels of the $e_{g}$ states decrease (increase), resulting in the decrease (increase) of the band gap. Clearly, the evolution
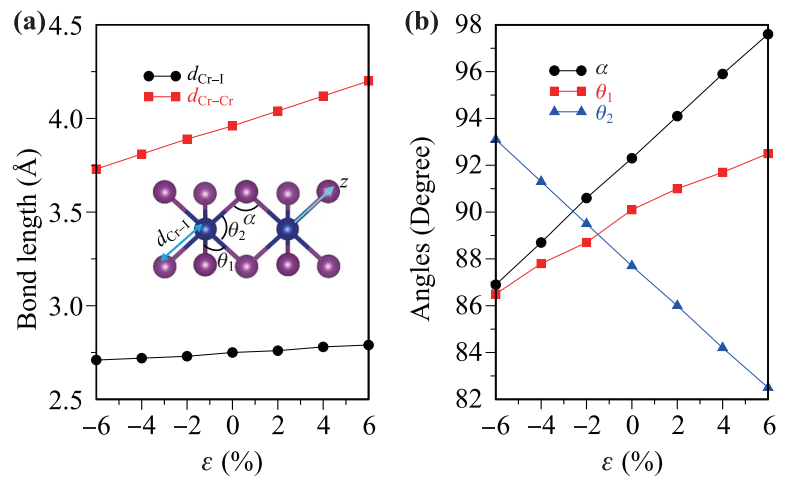

Fig. 3 Structure parameters of the AB-stacking bilayer $\mathrm{CrI}_{3}$ under biaxial strain. (a) The $\mathrm{Cr}-\mathrm{I}$ bond length $\left(d_{\mathrm{Cr}-\mathrm{I}}\right)$ and the distance between the nearest $\mathrm{Cr}$ atoms $\left(d_{\mathrm{Cr}-\mathrm{Cr}}\right)$. (b) Two types of the $\mathrm{I}-\mathrm{Cr}-\mathrm{I}$ angles and $\mathrm{Cr}-\mathrm{I}-\mathrm{Cr}$ angle. The $z$ axis of the local coordinate frame is indicated. of the band gap under external strain still accords with the trend shown in Fig. 4(a), if we consider the equivalent strain on the octahedron rather than the biaxial strain on the unit cell.

The biaxial strain also tunes the magnetic properties. As shown in Fig. 5, the local spin moment contributed by the $\mathrm{Cr}$ atoms $\left(M_{\mathrm{S}, \mathrm{Cr}}\right)$ increases as the strain change from $-6 \%$ to $6 \%$. In other words, the larger lattice constant results in the larger $M_{\mathrm{S}, \mathrm{Cr}}$. The total increment of $M_{\mathrm{S}, \mathrm{Cr}}$ is about $0.1 \mu_{B}$. However, the total spin moment of each $\mathrm{CrI}_{3}$ formula is independent of the strain and maintains $3.0 \mu_{B}$. It is clear that the value of $M_{\mathrm{S}, \mathrm{Cr}}$ is larger than the total spin moment, because the I atoms contribute about $-0.1 \mu_{B}$ per atom. Note that the stacking patterns do not (a)
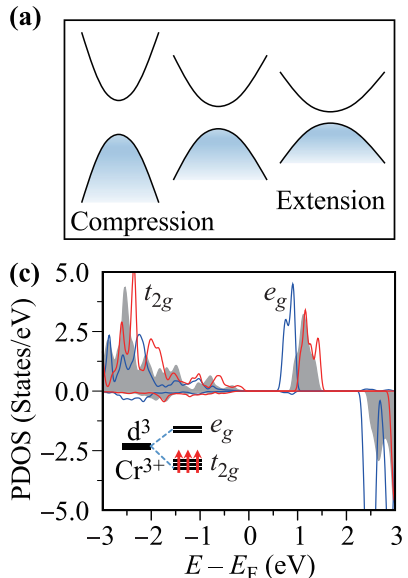

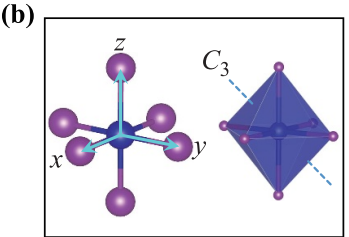

(d)

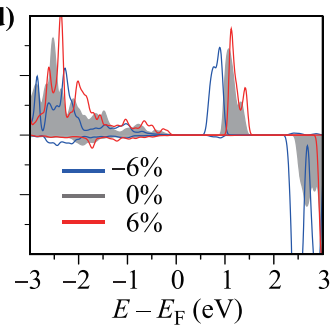

Fig. 4 (a) Sketch of the conventional evolution of the band structure under biaxial strain. (b) One $\mathrm{CrI}_{6}$ octahedron with the local coordinate frame. The dashed line indicates the $\mathrm{C}_{3}$ rotational axis and it is the direction of the equivalent strain on the octahedron to the biaxial strain on the unit cell. (c, d) Projected density of states (PDOS) of the 3d orbital of the $\mathrm{Cr}^{3+}$ ion under biaxial strains for the AB- and $\mathrm{AB}^{\prime}$ stacking bilayers, respectively. Positive (negative) sign refers to spin majority (minority) channel. The inset in (c) shows the schematic energy splitting of the $3 \mathrm{~d}$ orbitals of the $\mathrm{Cr}^{3+}$ ion. 


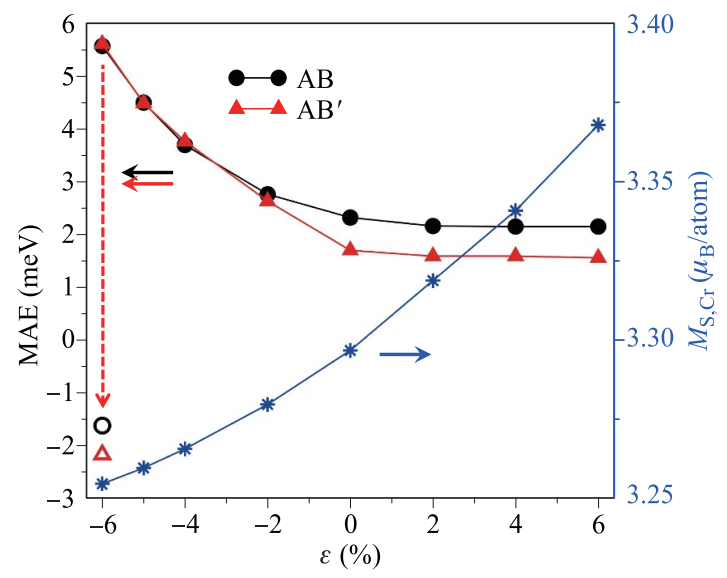

Fig. 5 Magnetic anisotropy energy (MAE) and local magnetic spin moment on $\mathrm{Cr}$ atom $\left(M_{\mathrm{S}, \mathrm{Cr}}\right)$ of the AB-stacking bilayer $\mathrm{CrI}_{3}$ under biaxial strain. The dashed arrow indicates the change of MAE when the FM-to-AFM transition occurs.

affect the spin moments. The magnetic anisotropy energy (MAE) are positive for all strains, indicating PMA for the bilayer $\mathrm{CrI}_{3}$, in agreement with experimental observations [4]. In addition, the MAE increases significantly when the amplitude of the compressive strain increases, while it decreases slightly when the extensile strain increases, similar with a recent report [26]. Furthermore, the MAE of the AB-stacking bilayer $\mathrm{CrI}_{3}$ is larger than that of the $\mathrm{AB}^{\prime}$-stacking bilayer $\mathrm{CrI}_{3}$ in most range of the strains. At the experimental lattice constant (i.e., $\varepsilon=$ $0)$, the MAEs of the AB- and $\mathrm{AB}^{\prime}$-stacking bilayers are $0.58 \mathrm{meV}$ and $0.34 \mathrm{meV}$ per $\mathrm{CrI}_{3}$ formula, respectively, in agreement with the recent report [23]. When $\varepsilon=-6 \%$, the MAE becomes $1.4 \mathrm{meV}$ per $\mathrm{CrI}_{3}$ formula for both $\mathrm{AB}$ - and $\mathrm{AB}^{\prime}$-stacking bilayers. Obviously, larger MAE guarantees better thermal stability at high temperature.

Note that the properties discussed above do not depend on the interlayer exchange interaction. The two $\mathrm{CrI}_{3}$ layers may couple with each other either ferromagnetically or antiferromagnetically [4]. The ground state can be determined by calculating the energy difference between the FM and AFM states: $\Delta E=E_{\mathrm{AFM}}-E_{\mathrm{FM}}$. As shown in Fig. 6(a), the AB-stacking bilayer $\mathrm{CrI}_{3}$ manifests strong interlayer ferromagnetism for the whole range of the strain. On the contrary, a FM-to-AFM transition occurs near $\varepsilon=-2 \%$ for the $\mathrm{AB}^{\prime}$-stacking bilayer $\mathrm{CrI}_{3}$. With compressive strain $\varepsilon \leq-2 \%$, the two $\mathrm{CrI}_{3}$ layers couple with each other ferromagnetically with the magnetic configuration in Fig. 2(a). The interlayer coupling becomes AFM for minor compressive strain and all extensile strain, and the corresponding magnetic configuration is similar with that plotted in Fig. 2(b). Therefore, the ground state of the $\mathrm{AB}^{\prime}$-stacking bilayer $\mathrm{CrI}_{3}$ is interlayer $\mathrm{AFM}$, in agreement with the experimental observation [4].

To reveal the underlying mechanism of the exchange couplings under the biaxial strain, we calculated the to-
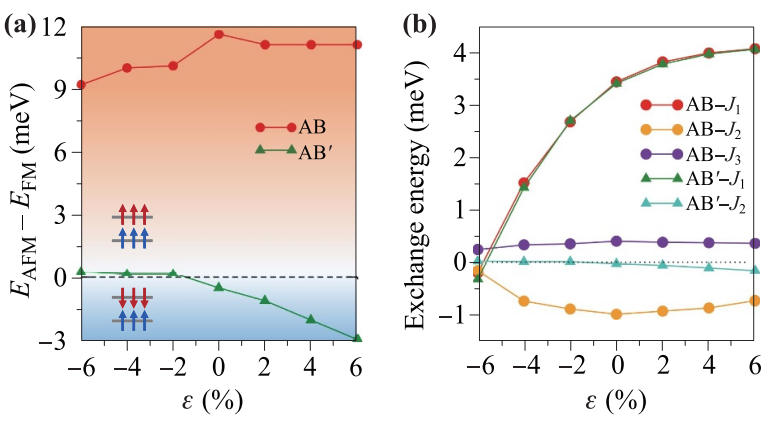

Fig. 6 (a) Energy difference between the interlayer FM and AFM states of the bilayer $\mathrm{CrI}_{3}$ under biaxial strain. (b) Exchange interaction energies between the $\mathrm{Cr}$ atoms as indicated in Fig. 1.

tal energies of five different magnetic configurations as plotted in Fig. 2. In the framework of Heisenberg model given by Eq. (1), the total energies related to the exchange couplings for the five different magnetic configurations in Fig. 2 for the AB-stacking bilayer $\mathrm{CrI}_{3}$ can be written as follows: $E_{a}=-\left(6 J_{1}+J_{2}+9 J_{3}\right) S^{2} ; E_{b}=-\left(6 J_{1}-J_{2}-\right.$ $\left.9 J_{3}\right) S^{2} ; E_{c}=-\left(J_{2}-3 J_{3}\right) S^{2} ; E_{d}=+\left(6 J_{1}+J_{2}-3 J_{3}\right) S^{2}$; $E_{e}=+\left(6 J_{1}-J_{2}+3 J_{3}\right) S^{2}$. For the $\mathrm{AB}^{\prime}$-stacking bilayer $\mathrm{CrI}_{3}$, the total energies are $E_{a}^{\prime}=-\left(6 J_{1}-4 J_{2}\right) S^{2} ; E_{b}^{\prime}=$ $-\left(6 J_{1}+4 J_{2}\right) S^{2} ; E_{c}^{\prime}=0 ; E_{d}^{\prime}=+6 J_{1} S^{2} ; E_{e}^{\prime}=+6 J_{1} S^{2}$. Here, $S=3 / 2$ is the spin quantum number of each $\mathrm{CrI}_{3}$ formula. Then we can obtain these exchange interaction parameters.

Figure 6(b) plots the exchange interaction parameters. It can be seen that the intralayer exchange interaction energy $\left(J_{1}\right)$ increases when the extensile strain increases and decreases when the compressive strain increases, for both $\mathrm{AB}$ - and $\mathrm{AB}^{\prime}$-stacking bilayers. The amplitudes of $J_{1}$ of the $\mathrm{AB}-$ and $\mathrm{AB}^{\prime}$-stacking bilayers are almost the same. In other words, the intralayer exchange interaction in bilayer $\mathrm{CrI}_{3}$ depends on the lattice constant monotonically but is independent of the stacking patterns. The larger lattice constant results in the larger $J_{1}$. In addition, the sign of $J_{1}$ changes around $\varepsilon=-6 \%$, which indicates a intralayer FM-to-AFM transition. Similar magnetic phase transition was also observed in the monolayer $\mathrm{CrI}_{3}[26,27]$. With the extensile strain $(\varepsilon>0)$ and moderate compressive strain $(-6 \%<\varepsilon<0)$, the neighboring $\mathrm{Cr}$ atoms in the same $\mathrm{CrI}_{3}$ layer couple with each other ferromagnetically. Combined the FM and AFM interlayer couplings respectively for the AB- and $\mathrm{AB}^{\prime}$-stacking bilayers, the $\mathrm{AB}$ - and $\mathrm{AB}^{\prime}$-stacking bilayers adopt the magnetic configurations displayed in Figs. 2(a) and (b), respectively, in this situation. With large compressive strain $(\varepsilon \leq-6 \%)$, the intralayer exchange interaction becomes AFM. Since the interlayer coupling is $\mathrm{FM}$ for both $\mathrm{AB}$ - and $\mathrm{AB}^{\prime}$-stacking bilayers, they adopt the magnetic configuration shown in Fig. 2(e). This magnetic configuration is the same as that reported recently [28]. The trend of the strain-dependent exchange interactions can be understood qualitatively as 
follows. It has been revealed that $J_{1}$ contains two parts of contributions: the direct exchange interaction between the first nearest neighboring $\mathrm{Cr}$ atom via $t_{2 g}-t_{2 g}$ orbitals and the indirect superexchange interaction via the $t_{2 g}-p$ $e_{g}$ orbitals [23-27]. Because of the octahedral crystal-field splitting, the former favors AFM exchange interaction, while the later favors FM exchange interaction. With extensive strain and moderate compressive strain, the indirect superexchange interaction dominates the intralayer exchange interaction in the $\mathrm{CrI}_{3}$ layer, which results in the FM ground state and positive $J_{1}$. Furthermore, when the lattice constant increases, the $\mathrm{Cr}-\mathrm{Cr}$ distance and the $\mathrm{Cr}-$ I-Cr angle ( $\alpha$ in Fig. 3 ) increases. Consequently, the direct exchange interaction is weakened, while the superexchange interaction is enhanced due to the stronger $t_{2 g}-p$ hybridization. These combined effects make $J_{1}$ increases. With large compressive strain $\varepsilon \leq-6 \%$, the $\mathrm{Cr}-\mathrm{Cr}$ distance is short enough, so that the $\mathrm{Cr}-\mathrm{Cr}$ direct exchange interaction dominates, resulting in AFM ground state and negative $J_{1}$. Moreover, when $\varepsilon=-6 \%$, the MAEs of the $\mathrm{AB}$ - and $\mathrm{AB}^{\prime}$-stacking bilayers are -1.60 and $-2.15 \mathrm{meV}$, respectively, as indicated by open symbols in Fig. 5, implying in-plane MA rather than PMA.

For the interlayer exchange interactions of the $\mathrm{AB}$ stacking bilayer $\mathrm{CrI}_{3}, J_{2}$ is negative and its amplitudes in the most range of the strain are large, indicating that the two Cr atoms connected by $J_{2}$ (see Fig. 1) prefer AFM coupling. $J_{3}$ is positive and its amplitude is smaller than that of $J_{2}$. For each $\mathrm{Cr}$ atom, there is only one pair of exchange interaction represented by $J_{2}$, while there are six pairs of exchange interaction represented by $J_{3}$. Therefore, the final interlayer exchange interaction is FM. For the $\mathrm{AB}^{\prime}$-stacking bilayer $\mathrm{CrI}_{3}$, the amplitudes of $J_{2}$ are relatively small, and the sign changes around $\varepsilon=-2 \%$, corresponding to the FM-to-AFM transition as shown in Fig. 6(a).

With the exchange interaction parameters, we carried out Monte Carlo (MC) simulation based on the Heisenberg model as expressed in Eq. (1). Although the bulk $\mathrm{CrI}_{3}$ is believed to be Ising type FM magnet [4], we found that the Ising model overestimates $T_{\mathrm{C}}$ greatly. For the monolayer $\mathrm{CrI}_{3}$, the Curie temperatures from the Ising model, Heisenberg model, and mean-field theory are $130 \mathrm{~K}, 90 \mathrm{~K}$ and $52 \mathrm{~K}$, respectively. Apparently, only the mean-field theory predicts proper $T_{\mathrm{C}}$ close to the experiment measurement $(45 \mathrm{~K})$. Nevertheless, the mean-field theory could not be applied to the bilayer $\mathrm{CrI}_{3}$ due to the complicated exchange interactions. Therefore, we choose the Heisenberg model to estimate the Curie temperatures of the bilayer $\mathrm{CrI}_{3}$ under biaxial strains. Although the amplitudes of the Curie temperatures are expected to be overestimated, qualitative investigation of the response of $T_{\mathrm{C}}$ to the biaxial strain is still meaningful.

Figure 7(a) plots the magnetization (i.e., the average spin moment per $\mathrm{CrI}_{3}$ formula) as a function of temperature for the $\mathrm{AB}^{\prime}$-stacking bilayer $\mathrm{CrI}_{3}$, from the $\mathrm{MC}$ sim-
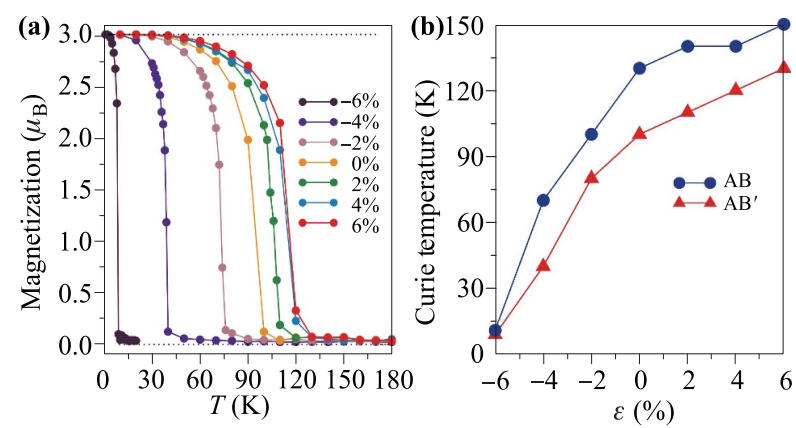

Fig. 7 (a) Magnetization (average spin moment per $\mathrm{CrI}_{3}$ formula) of the $\mathrm{AB}^{\prime}$-stacking bilayer $\mathrm{CrI}_{3}$ at different temperatures. (b) Curie temperature of the bilayer $\mathrm{CrI}_{3}$ under biaxial strain.

ulations. It can be seen that the average spin moment sharply decreases from $3 \mu_{B}$ to 0 at certain temperature for each strain, corresponding to the $T_{\mathrm{C}}$. The Curie temperatures of the strain-free $\mathrm{AB}$ - and $\mathrm{AB}^{\prime}$-stacking bilayers increase to about $130 \mathrm{~K}$ and $100 \mathrm{~K}$ (i.e., at $\varepsilon=0$ ), respectively, which means that both the interlayer FM exchange interaction in the AB-stacking bilayer $\mathrm{CrI}_{3}$ and the interlayer $\mathrm{AFM}$ exchange interaction in the $\mathrm{AB}^{\prime}$-stacking bilayer $\mathrm{CrI}_{3}$ have positive effect on the $\mathrm{FM}$ ordering, compared to the monolayer $\mathrm{CrI}_{3}$. With the strain varing from $-6 \%$ to $6 \%$, the $T_{\mathrm{C}}$ increases from $11 \mathrm{~K}$ to $160 \mathrm{~K}$ for the AB-stacking bilayer $\mathrm{CrI}_{3}$ and from $9 \mathrm{~K}$ to $130 \mathrm{~K}$ for the $\mathrm{AB}^{\prime}$-stacking bilayer $\mathrm{CrI}_{3}$, as shown in Fig. 7(b). This trend is similar to that of $J_{1}$, indicating that the $T_{\mathrm{C}}$ is mainly determined by the intralayer exchange interactions in the bilayer $\mathrm{CrI}_{3}$, similar to the monolayer $\mathrm{CrI}_{3}$. The FM ordering in the AB-stacking bilayer $\mathrm{CrI}_{3}$ is further enhanced by the interlayer exchange interactions, which leads to higher $T_{\mathrm{C}}$ with respect to the $\mathrm{AB}^{\prime}$-stacking bilayer $\mathrm{CrI}_{3}$ at the same strain. It is worth noting that the stability of the magnetic ordering depends on not only the $T_{\mathrm{C}}$ but also the MAE. Although the MAEs of the bilayer $\mathrm{CrI}_{3}$ under extensile biaxial strain decrease slightly relative to strain-free case (see Fig. 5), the MAEs are already large enough to ensure the magnetic ordering bellow Curie temperatures for both $\mathrm{AB}$ - and $\mathrm{AB}^{\prime}$-stacking bilayers.

Expect for the biaxial strain, the hydrostatic pressure may also modify the magnetism of the bilayer $\mathrm{CrI}_{3}[20,21]$. Because the interlayer distance is much easier to be changed than the lateral lattice constant, the hydrostatic pressure can be regarded as the compressive strain on the octahedron along the $C_{3}$ axis as denoted in Fig. 4(b). Here, we use the decrement of the distance between the two $\mathrm{CrI}_{3}$ layers $(\Delta d)$ to represent the hydrostatic pressure. We firstly calculated the energy difference between the interlayer FM and AFM states, as plotted in Fig. 8(a). For the AB-stacking bilayer $\mathrm{CrI}_{3}, \Delta E$ keeps positive and increases rapidly as $\Delta d$ increases, which indicates that the interlayer exchange is strongly FM. This can also be manifested by the rapidly increasing $J_{2}$ and positive $J_{3}$ 

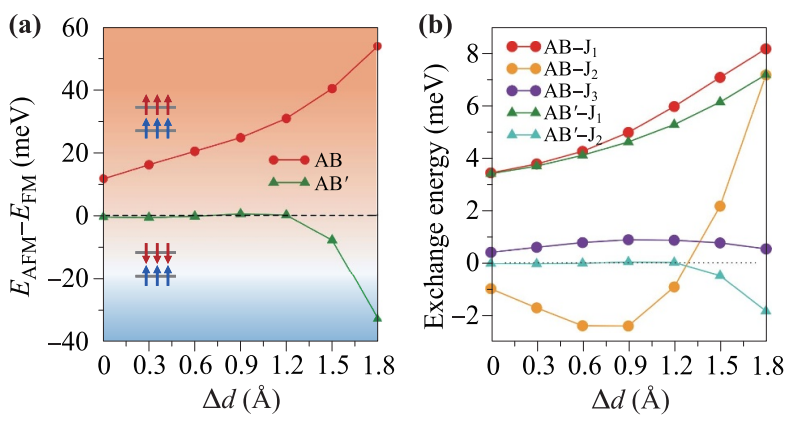

Fig. 8 (a) Energy difference between the interlayer FM and AFM states of the bilayer $\mathrm{CrI}_{3}$ under hydrostatic pressure. (b) Exchange interaction energies between the $\mathrm{Cr}$ atoms as indicated in Fig. 1.

as shown in Fig. 8(b). For the $\mathrm{AB}^{\prime}$-stacking bilayer $\mathrm{CrI}_{3}$, $\Delta E$ increases slightly first, then decreases strikingly when $\Delta d>1.2 \AA$. In addition, the sign of $\Delta E$ at $\Delta d=0.9$ and $1.2 \AA$ is positive, implying that the $\mathrm{AB}^{\prime}$-stacking bilayer $\mathrm{CrI}_{3}$ favors the interlayer AFM state at this situation.

The intralayer exchange interactions in both $\mathrm{AB}$ - and $\mathrm{AB}^{\prime}$-stacking bilayers remain $\mathrm{FM}$, as manifested by the positive values of $J_{1}$ in Fig. 8(b). Moreover, $J_{1}$ increases monotonically as $\Delta d$ increases for both systems, and the magnitudes of $J_{1}$ are much larger than the others at the same biaxial strain. Therefore, the Curie temperatures increase significantly up to $320 \mathrm{~K}$ and $280 \mathrm{~K}$ respectively for the $\mathrm{AB}$ - and $\mathrm{AB}^{\prime}$-stacking bilayers, as shown in Fig. 9(a). Although $T_{\mathrm{C}}$ is overestimated by the Heisenberg model, the relative enlargement could be instructive. Considering that the amplitudes of $T_{\mathrm{C}}$ of the $\mathrm{AB}$ - and $\mathrm{AB}^{\prime}$-stacking bilayers have been enlarged by about 2.5 times at $\Delta d=$ $1.8 \AA$ compared to those at pressure-free systems, $T_{\mathrm{C}}$ over $100 \mathrm{~K}$ could be expected in real experimental measurement.

The MAE of the AB-stacking bilayer $\mathrm{CrI}_{3}$ decreases monotonically as $\Delta d$ increases, as shown in Fig. 9(b). For the $\mathrm{AB}^{\prime}$-stacking bilayer $\mathrm{CrI}_{3}$, the MAE decreases first,
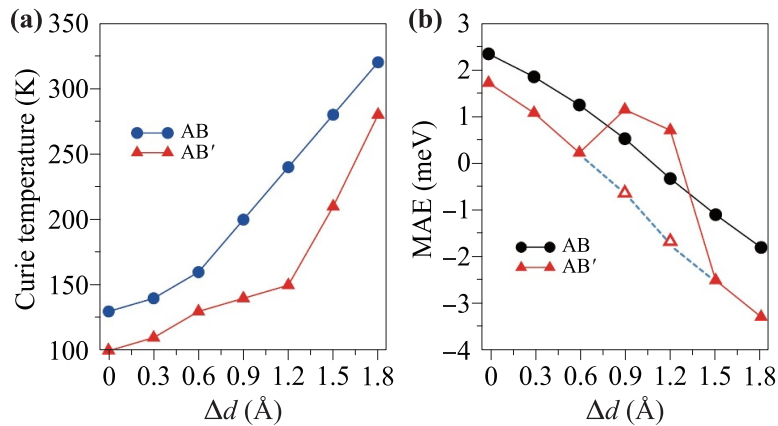

Fig. 9 (a) Curie temperature and (b) magnetic anisotropy energy (MAE) of the bilayer $\mathrm{CrI}_{3}$ under hydrostatic pressure. The open triangles were calculated with interlayer AFM state for the $\mathrm{AB}^{\prime}$-stacking bilayer $\mathrm{CrI}_{3}$. then increases at $\Delta d=0.9 \AA$, and decreases again when $\Delta d>0.9 \AA$. In fact, the $\mathrm{AB}^{\prime}$-stacking bilayer $\mathrm{CrI}_{3}$ favors the interlayer FM state at $\Delta d=0.9$ and $1.2 \AA$ as seen in Fig. 8(a), so that the MAEs behave differently from the other points. If the $\mathrm{AB}^{\prime}$-stacking bilayer $\mathrm{CrI}_{3}$ is in the interlayer AFM state, the MAEs follow the trend formed by the other points, as manifested by the open triangles and connected by the blue dashed lines in Fig. 9(b). When $\Delta d>1.5 \AA$, the MAEs of both AB- and $\mathrm{AB}^{\prime}$-stacking bilayers are negative, indicating in-plane MA. Nonetheless, the amplitudes of the MAEs are comparable to or even larger than those of the press-free systems, especially for $\Delta d=1.8 \AA$, hence the magnetic ordering could still be retained bellow $T_{\mathrm{C}}$.

\section{Conclusions}

In summary, we investigated the electronic and magnetic properties of two types of bilayer $\mathrm{CrI}_{3}$ under biaxial strain and hydrostatic pressure, based on first-principles calculations and Monto Carlo simulation. We found that the band gaps, MAEs, intralayer and interlayer exchange interactions, and Curie temperatures can be tuned by the biaxial strain and hydrostatic pressure. The band gap decreases (increases) under compressive (extensile) strain, which is related to the symmetry of the $\mathrm{CrI}_{6}$ octahedron. The MAE increases under compressive strain, but decreases under extensile strain and hydrostatic pressure. The interlayer FM coupling of the AB-stacking bilayer $\mathrm{CrI}_{3}$ is robust under both biaxial strain and hydrostatic pressure. The interlayer coupling of the $\mathrm{AB}^{\prime}$-stacking bilayer $\mathrm{CrI}_{3}$ is sensitive to the biaxial strain. It prefers the interlayer AFM state under extensile strain and small compressive strain $(\varepsilon>-2 \%)$, but turns to the interlayer FM state for larger compressive stain. For both bilayers, the intralayer magnetic ordering may undergo a FM-to-AFM transition under large compressive strain $(\varepsilon<-6 \%)$. The interlayer exchange interaction may be significantly enhanced by the hydrostatic pressure, which may boost the Curie temperature over $100 \mathrm{~K}$.

Acknowledgements This work was supported by the National Natural Science Foundation of China (No. 11574223) and the Six Talent Peaks Project of Jiangsu Province (No. 2019-XCL-081).

\section{References}

1. 1. K. S. Novoselov, A. K. Geim, S. V. Morozov, D. Jiang, Y. Zhang, S. V. Dubonos, I. V. Grigorieva, and A. A. Firsov, Electric field effect in atomically thin carbon films, Science 306(5696), 666 (2004)

2. X. M. Li, L. Tao, Z. F. Chen, H. Fang, X. S. Li, X. R. Wang, J. B. Xu, and H. W. Zhu, Graphene and related two-dimensional materials: Structure-property relation- 
ships for electronics and optoelectronics, Appl. Phys. Rev. 4(2), 021306 (2017)

3. C. Gong, L. Li, Z. Li, H. Ji, A. Stern, Y. Xia, T. Cao, W. Bao, C. Wang, Y. Wang, Z. Q. Qiu, R. J. Cava, S. G. Louie, J. Xia, and X. Zhang, Discovery of intrinsic ferromagnetism in two-dimensional van der Waals crystals, Nature 546(7657), 265 (2017)

4. B. Huang, G. Clark, E. Navarro-Moratalla, D. R. Klein, R. Cheng, K. L. Seyler, D. Zhong, E. Schmidgall, M. A. McGuire, D. H. Cobden, W. Yao, D. Xiao, P. JarilloHerrero, and X. D. Xu, Layer-dependent ferromagnetism in a van der Waals crystal down to the monolayer limit, Nature 546(7657), 270 (2017)

5. N. D. Mermin and H. Wagner, Absence of ferromagnetism or anti-ferromagnetism in one- or two-dimensional isotropic Heisenberg models, Phys. Rev. Lett. 17(22), 1133 (1966)

6. M. Bonilla, S. Kolekar, Y. Ma, H. C. Diaz, V. Kalappattil, R. Das, T. Eggers, H. R. Gutierrez, M. H. Phan, and M. Batzill, Strong room-temperature ferromagnetism in $\mathrm{VSe}_{2}$ monolayers on van der Waals substrates, Nat. Nanotechnol. 13(4), 289 (2018)

7. Y. Deng, Y. Yu, Y. Song, J. Zhang, N. Z. Wang, Z. Sun, Y. Yi, Y. Z. Wu, S. Wu, J. Zhu, J. Wang, X. H. Chen, and Y. B. Zhang, Gate-tunable room-temperature ferromagnetism in two-dimensional $\mathrm{Fe}_{3} \mathrm{GeTe}_{2}$, Nature 563(7729), 94 (2018)

8. C. Gong and X. Zhang, Two-dimensional magnetic crystals and emergent heterostructure devices, Science 363(6428), eaav4450 (2019)

9. B. Huang, M. A. McGuire, A. F. May, D. Xiao, P. JarilloHerrero, and $\mathrm{X}$. D. Xu, Emergent phenomena and proximity effects in two-dimensional magnets and heterostructures, Nat. Mater. 19(12), 1276 (2020)

10. J. F. Jr Dillon and C. E. Olson, Magnetization, resonance, and optical properties of the ferromagnet $\mathrm{CrI}_{3}, J$. Appl. Phys. 36(3), 1259 (1965)

11. M. A. McGuire, H. Dixit, V. R. Cooper, and B. C. Sales, Coupling of crystal structure and magnetism in the layered, ferromagnetic insulator $\mathrm{CrI}_{3}$, Chem. Mater. 27(2), 612 (2015)

12. T. Latychevskaia, S. K. Son, Y. Yang, D. Chancellor, M. Brown, S. Ozdemir, I. Madan, G. Berruto, F. Carbone, A. Mishchenko, and K. S. Novoselov, Stacking transition in rhombohedral graphite, Front. Phys. 14, 13608 (2019)

13. N. Sivadas, S. Okamoto, X. D. Xu, C. J. Fennie, and D. Xiao, Stacking-dependent magnetism in bilayer $\mathrm{CrI}_{3}$, Nano Lett. 18(12), 7658 (2018)

14. P. H. Jiang, C. Wang, D. C. Chen, Z. C. Zhong, Z. Yuan, Z. Y. Lu, and W. Ji, Stacking tunable interlayer magnetism in bilayer $\mathrm{CrI}_{3}$, Phys. Rev. B 99(14), 144401 (2019)

15. D. Soriano, C. Cardoso, and J. Fernández-Rossier, Interplay between interlayer exchange and stacking in $\mathrm{CrI}_{3}$ bilayers, Solid State Commun. 299, 113662 (2019)

16. S. W. Jang, M. Y. Jeong, H. Yoon, S. Ryee, and M. J. Han, Microscopic understanding of magnetic interactions in bilayer $\mathrm{CrI}_{3}$, Phys. Rev. Mater. 3(3), 031001 (2019)
17. S. Jiang, J. Shan, and K. F. Mak, Electric-field switching of two-dimensional van der Waals magnets, Nat. Mater. 17(5), 406 (2018)

18. B. Huang, G. Clark, D. R. Klein, D. MacNeill, E. Navarro-Moratalla, K. L. Seyler, N. Wilson, M. A. McGuire, D. H. Cobden, D. Xiao, W. Yao, P. JarilloHerrero, and $\mathrm{X}$. D. Xu, Electrical control of 2D magnetism in bilayer $\mathrm{CrI}_{3}$, Nat. Nanotechnol. 13(7), 544 (2018)

19. X. X. Zhang, L. Li, D. Weber, J. Goldberger, K. F. Mak, and J. Shan, Gate-tunable spin waves in antiferromagnetic atomic bilayers, Nat. Mater. 19(8), 838 (2020)

20. T. Li, S. Jiang, N. Sivadas, Z. Wang, Y. Xu, D. Weber, J. E. Goldberger, K. Watanabe, T. Taniguchi, C. J. Fennie, K. Fai Mak, and J. Shan, Pressure-controlled interlayer magnetism in atomically thin $\mathrm{CrI}_{3}$, Nat. Mater. 18(12), 1303 (2019)

21. T. C. Song, Z. Y. Fei, M. Yankowitz, Z. Lin, Q. N. Jiang, K. Hwangbo, Q. Zhang, B. S. Sun, T. Taniguchi, K. Watanabe, M. A. McGuire, D. Graf, T. Cao, J. H. Chu, D. H. Cobden, C. R. Dean, D. Xiao, and X. D. Xu, Switching 2D magnetic states via pressure tuning of layer stacking, Nat. Mater. 18(12), 1298 (2019)

22. J. L. Lado and J. Fernández-Rossier, On the origin of magnetic anisotropy in two dimensional $\mathrm{CrI}_{3}, 2 D$ Mater. 4, 035002 (2017)

23. F. Xue, Y. Hou, Z. Wang, and R. Wu, Two-dimensional ferromagnetic van der Waals $\mathrm{CrCl}_{3}$ monolayer with enhanced anisotropy and Curie temperature, Phys. Rev. B 100(22), 224429 (2019)

24. J. Liu, P. Mo, M. Shi, D. Gao, and J. Lu, Multiscale analysis of strain-dependent magnetocrystalline anisotropy and strain-induced Villari and NagaokaHonda effects in a two-dimensional ferromagnetic chromium tri-iodide monolayer, J. Appl. Phys. 124(4), 044303 (2018)

25. J. Liu, M. Shi, J. Lu, and M. P. Anantram, Analysis of electrical-field-dependent Dzyaloshinskii-Moriya interaction and magnetocrystalline anisotropy in a twodimensional ferromagnetic monolayer, Phys. Rev. B 97(5), 054416 (2018)

26. L. Webster and J. A. Yan, Strain-tunable magnetic anisotropy in monolayer $\mathrm{CrCl}_{3}, \mathrm{CrBr}_{3}$, and $\mathrm{CrI}_{3}$, Phys. Rev. B 98(14), 144411 (2018)

27. F. Zheng, J. Zhao, Z. Liu, M. Li, M. Zhou, S. Zhang, and P. Zhang, Tunable spin states in the two-dimensional magnet $\mathrm{CrI}_{3}$, Nanoscale 10(29), 14298 (2018)

28. A. M. León, J. W. González, J. Mejía-López, F. Crasto de Lima, and E. S. Morell, Strain-induced phase transition in $\mathrm{CrI}_{3}$ bilayers, 2D Mater. 7, 035008 (2020)

29. G. Kresse and J. Furthmüller, Efficiency of ab-initio total energy calculations for metals and semiconductors using a plane-wave basis set, Comput. Mater. Sci. 6(1), 15 (1996)

30. G. Kresse and J. Furthmüller, Efficient iterative schemes for ab initio total-energy calculations using a plane-wave basis set, Phys. Rev. B 54(16), 11169 (1996)

31. P. E. Blöchl, Projector augmented-wave method, Phys. Rev. B 50(24), 17953 (1994) 
32. G. Kresse and D. Joubert, From ultrasoft pseudopotentials to the projector augmented-wave method, Phys. Rev. B 59(3), 1758 (1999)

33. J. P. Perdew, K. Burke, and M. Ernzerhof, Generalized gradient approximation made simple, Phys. Rev. Lett. 77(18), 3865 (1996)
34. J. Klimeš, D. R. Bowler, and A. Michaelides, Van der Waals density functionals applied to solids, Phys. Rev. B 83(19), 195131 (2011)

35. A. I. Liechtenstein, V. I. Anisimov, and J. Zaanen, Density-functional theory and strong interactions: Orbital ordering in Mott-Hubbard insulators, Phys. Rev. B 52(8), R5467 (1995) 